asked ourselves if the genetic associations would differ with the new cluster stratification.

Methods For drug analysis we used two described sets of patients (1) selecting gene expression data of one visit/patient with active SLE (SLEDAI>5). Patient gene signatures were compared to drug derived gene signatures from CLUE database, giving a connectivity score. A negative score reflects inverse patterns between two signatures implying the drug may revert the disease-signature while a positive score would simulate disease. The magnitude of the score reflects the potential efficacy of the drug. Genetic data was performed in independent sets of individuals, focusing on the HLA.

Results Patient stratification based on drug connectivity scores revealed the same cluster structure described (correlation between neutrophil/lymphocyte ratio and SLEDAI dNLR $\mathrm{p}=1 \times 10-7$ ), implying that differential treatment depends on the cluster to which patients belong. Although drugs commonly used in SLE did not show the best scores, we found different values for each cluster suggesting that expression of target genes may provide insight in the prioritization of compounds.

We next constructed a model to classify patients using cluster information to inform on drug use and predict nephritis applied to 3 new longitudinal cohorts. A meta-analysis showed a significantly higher incidence of nephritis in patients classified to a neutrophil-driven cluster (2). In addition we observed differences in the genetic associations to disease in the HLA region depending on the clusters.

Conclusions Drug patterns reverting disease gene expression follow the cell-specificity of the disease clusters and provide a clinically useful model to treatment selection and nephritis. Clustering, at least in one case is also guided by the genetic contribution to disease.

\section{$14 \quad$ PLANNING AND MANAGEMENT OF LUPUS NEPHRITIS IN PREGNANCY}

\section{David P D'Cruz. Louise Coote Lupus Unit, Guy's and St Thomas' Hospitals, London UK}

\subsection{6/lupus-2020-eurolupus.4}

Background SLE primarily affects young women in their childbearing years and lupus nephritis is a common complication that often develops early in the disease course. Patients with lupus nephritis are at increased risk of fetal and maternal morbidity.

Methods Careful pre-pregnancy counselling is essential and should involve a multidisciplinary team with experience of managing high risk pregnancies. Careful management of active lupus nephritis aiming for complete clinical remission and optimising treatment to safely discontinue fetotoxic therapies is essential prior to discussing pregnancy. While these aspects are being managed, effective contraception is vital to avoid unplanned pregnancy with active lupus nephritis. The broader picture including previous obstetric history, assessment for antiphospholipid antibodies and neonatal lupus syndrome in Ro/La positive women also needs to be considered. Patients with lupus nephritis, even when in remission need very close monitoring throughout pregnancy and the post-natal period.

Results Data in the literature suggests that lupus flares including lupus nephritis flares, hypertension and pre-eclampsia may occur in up to $25 \%$ of patients. Lupus nephritis flares in pregnancy increase the risk of renal impairment, intra-uterine growth restriction, premature delivery and fetal morbidity including stillbirths and neonatal deaths and rarely maternal mortality.

Conclusions With careful management, women with lupus nephritis can have successful pregnancies.

\section{DEFINING LUPUS: CHANCES AND CHALLENGES BASED ON THE EULAR/ACR CRITERIA}

Martin Aringer. Division of Rheumatology, Department of Medicine III, University Medical Center and Faculty of Medicine TU Dresden, Dresden, Germany

\subsection{6/lupus-2020-eurolupus.5}

Background The EULAR/ACR classification criteria for SLE have introduced three new concepts. One, ANA, with their high sensitivity, but low specificity, were re-positioned to an entry criterion. Two, all criteria items are now attributed specific weights, and this often means that two criteria, such as arthritis and antibodies to double-stranded DNA (dsDNA), are sufficient to reach the 10 point classification line. Three, instead of long lists of exclusion criteria, there is one attribution rule for all items, namely that criteria are only to be counted for SLE if there is no more likely alternative cause such as rheumatoid arthritis for arthritis in an anti-CCP positive patient. All these concepts have individually led to discussions and misunderstandings.

Results Although ANA need to be positive only once ever, it is clear that this entry criterion still excludes patients who were always ANA negative. ANA negative SLE exists, but is uncommon, which makes this much less of a problem for classification than for diagnosis, and it is important to re-iterate that the diagnosis must be possible independent of classification criteria. In poor countries, ANA testing may be a problem, but worldwide availability of such a test must be an obvious goal. Test quality has also been discussed. Critically, not all ANA substrates had adequate sensitivity, and this has to be resolved.

For the lupus specific antibodies to Sm and dsDNA, with a relative weight of 6 points, specificity is the more critical issue. Many anti-dsDNA tests have insufficient test specificity, so that anti-dsDNA can only be counted if positive in a test with at least $90 \%$ specificity against appropriate disease controls. This is typically true for Crithidia and Farr assays, but not for many of the high throughput tests of today. Clinicians will have to be aware of the test characteristics of the antidsDNA tests they have available. SLE classification needs knowledge of autoantibody testing, and appropriate training of physicians will be key.

The attribution rule probably is the most challenging concept. In essence, criteria should not be counted for the classification of SLE if not accepted for expert diagnosis. In this sense, attribution is very close to the clinical process of diagnosis, as hoped for, but this needs knowledge on typical findings of other autoimmune diseases that are relevant differential diagnoses to SLE, such as primary anti-phospholipid syndrome or Sjögren's syndrome. Appropriate attribution may be troublesome in data bases, and not following the attribution rule with diligence will automatically diminish the specificity of the new criteria. Above attribution, a misdiagnosis of for example mucocutaneous items, such as mistaking Rosacea for malar rash, will also cause trouble. Again, appropriate training will be necessary. 
Despite these potential pitfalls, we believe that the EULAR/ ACR criteria are a relevant step forward in appropriately defining who has SLE, and in teaching doctor and medical students how to approach a patient with possible SLE. The data have clearly demonstrated that ANA negative SLE is uncommon, and ANA are a useful entry criterion or screening parameter in case of suspected SLE. The analysis of interaction has important implications in that it has shown interactions within domains, upholding this concept, but not found significant associations between domains (or items in various domains). The latter in fact is an argument that SLE is indeed a disease, not a syndrome, and that it is the effector arm of the autoantibodies in any given SLE patient that underlies the variability.

Conclusions We will need more knowledge on autoantibodies, not less, and probably more clinical training, but this is more of a chance than a challenge. Above all, it has been remarkably easy to work together in this huge group, over the Atlantic and beyond, and I am deeply gratefully for the contribution of so many colleagues. This large team experience of collegiality and friendship will hopefully help the further worldwide collaboration that is necessary for advancing the field.

Acknowledgements The EULAR/ACR SLE classification project was equally supported by EULAR and ACR. Please refer to the publications for contributors to this effort.

\section{THE HISTORY OF LUPUS THROUGHOUT THE AGES}

Laurent Arnaud. Dept. of rheumatology, National French Reference Center for Auto-immune Diseases (RESO), INSERM 1109, Institut PACIFIC, Strasbourg, France

\subsection{6/lupus-2020-eurolupus.6}

The word lupus (Latin term for the wolf) is mentioned for the first time circa $850 \mathrm{AD}$. Originally, the term was used to describe lesions that were said to resemble wolves' bite. Early historical documents have suggested that the term lupus was used indistinctively during the middle-age and the Renaissance for many types of diseases characterized by ulcerous lesions, especially in the lower limbs. In 1230, Rolando of Parma distinguishes 'noli me tangere' (lesions located on the face) from lupula when the lesions are on the limbs. Four centuries later, there is still a great deal of confusion with Sennert, Culpeper \& Cole who mentioned (1661) that '[...] cancer can be divided into 3 species: into the cancer, [...] the noli me tangere $[\ldots]$ and lupus or the wolf if it is in the shins, ankle-bones and thighs'.

The true turning point in the history of lupus occurred in London in the beginning of the 19th century when Robert Willan and his student Bateman published the first atlases of skin diseases (1786-1817), containing the first known representation of a patient with lupus (figure 1). At that time, the disease was classified among 'tubercula' and the distinction between lupus vulgaris (skin tuberculosis) and cutaneous lupus in its modern sense was not clearly made until Keil in 1933. In 1850, the French dermatologist Cazenave mentions for the first time the term 'lupus érythemateux'. Kaposi describes the 'butterfly rash' in 1872 and identifies discoid lupus as a separate entity in 1875 . The history of lupus will take a new turn between 1872 and 1905, with major contributions from Kaposi, Sequiera \& Balean, and Osler who will enable the true recognition of the systemic nature of the disease

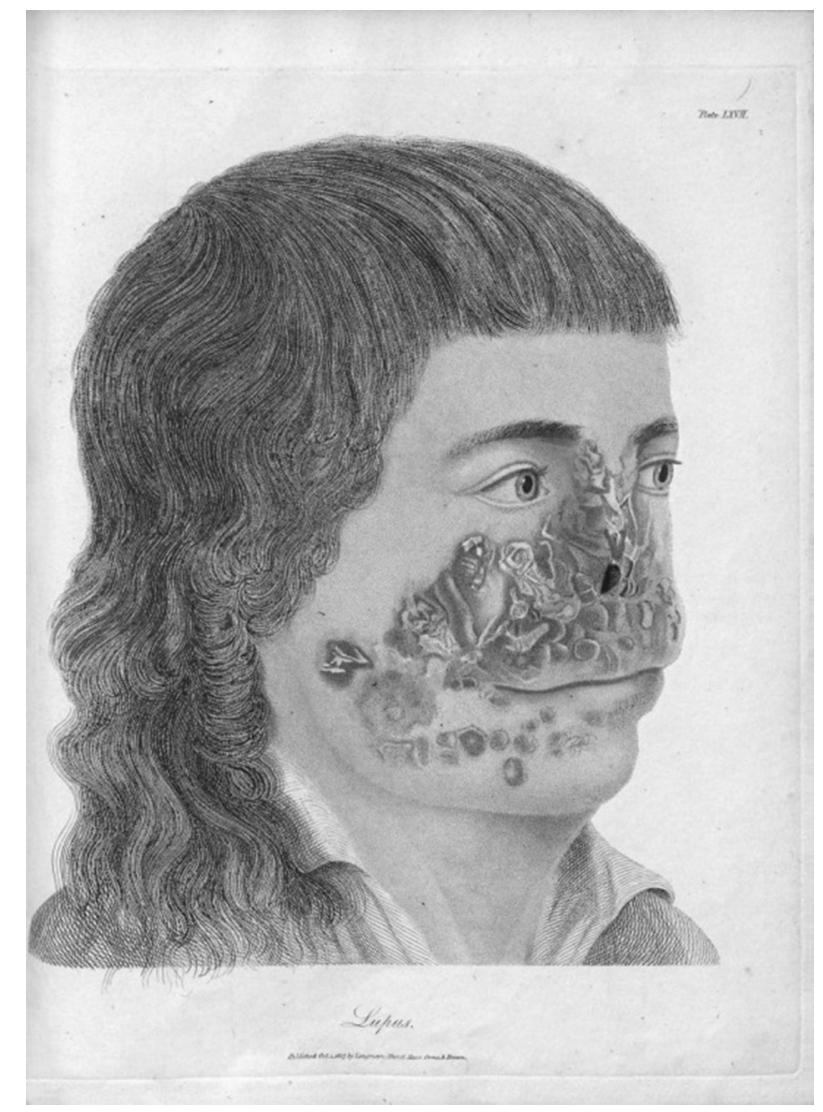

Abstract 16 Figure 1 Delineations of cutaneous diseases. Thomas Bateman, London (1918)

(Systemic Lupus Erythematosus). The modern history of lupus is notably marked by the discovery of lupus cells (LE cells) by Hargraves in 1948, of antinuclear antibodies by Miescher in 1954 and by the recognition of DNA as the main target of ANAs by Seligman in 1957.

Many treatments have been proposed for lupus throughout the ages, including the use of cauterization \& caustics (from the middle age to the modern era), radium (1900-1905), and even concentrated sun light \& UVs in London in 1905! Quinine was introduced in 1894 while most modern treatments for SLE appeared in the second half of the 20th century: glucocorticoids (1948-1952), quinacrine (1951), cyclophosphamide (1954), hydroxychloroquine (1956), azathioprine (1957) and mycophenolate mofetil (1980s). The end of the 20th century and the beginning of the 21st century are marked by a better understanding of the pathogenesis of the disease and the systematic evaluation of treatments, paving the way for improved diagnosis and better care for lupus patients.

\section{PATIENTS EXPECTATIONS, AND WHAT WE (CAN) DO ABOUT IT}

Alain Cornet, Kirsi Myllys, Anne Charlet, Annemarie Sluijmers, Marisa Costa, Elfried Wijsma, Jeanette Andersen. Lupus Europe, UK

\subsection{6/lupus-2020-eurolupus.7}

Background Lupus patient's expectations are no different than everyone else's: 'A better life'. But achieving it requires different steps because it entails (1) A prompt diagnosis, (2) Access to effective treatment with low side-effects, (3) Resolving 